Veronica Marie M. Mendoza, MD Juan Ramon V. Perez De Tagle, MD Adrian F. Fernando, MD

\section{Department of Otorhinolaryngology} Head and Neck Surgery University of Santo Tomas Hospital
Correspondence: Dr. Adrian F. Fernando Department of Otorhinolaryngology Head and Neck Surgery University of Santo Tomas Hospital España Boulevard, Manila 1108 Philippines

Phone: (632) 7313001 local 2478

Email: ianfernando_md@yahoo.com

The authors declared that this represents original material, that the manuscript has been read and approved by the authors, that the requirements for authorship have been met by the authors, and that the authors believe that the manuscript represents honest work

Disclosures: The authors signed a disclosure that there are no financial or other (including personal) relationships, intellectual passion, political or religious beliefs, and institutional affiliations that might lead to a conflict of interest.

Presented at the 2nd International Congress Free Paper Presentation for Medical Sciences, January 16, 2016. St. Paul University, Tugegarao City, Philippines.

Institutional Review Board approval and written informed consent for publication were obtained for this Case Report.

\section{(1) $(\Theta \Theta$}

Creative Commons (CC BY-NC-ND 4.0)

Attribution - NonCommercial - NoDerivatives 4.0 International

\section{Peripheral T-Cell Lymphoma Presenting as an Auricle Mass}

When evaluating patients with a non-traumatic auricular deformity that presents like a soft tissue infection unresponsive to antibiotic therapy and progressively resembles a tumor, immediate biopsy and imaging should be instituted to obtain an accurate diagnosis and avoid unnecessary procedures. After all, not all head and neck masses are managed with surgery.

\section{CASE REPORT}

A 64-year-old diabetic man with a 30-pack/year smoking history presented with progressive diffuse swelling of the right auricule with exudative yellow non-foul smelling discharge. Initially diagnosed with auricular cellulitis by an ENT specialist, there was no response to oral and topical antibiotic treatment as the swelling developed into a large cauliflower-like deformity. Incisional biopsy only revealed fibrocollagenous tissue with chronic inflammation without evident granuloma.

With the progressively enlarging right auricular mass unresponsive to medical treatment for over six months, the man underwent a series of multi-disciplinary consultations in our institution. Also noted were left tragal enlargement without ulceration or bleeding, palpable level II-III cervical lymph nodes (measuring $2.5 \mathrm{~cm}$ in widest diameter), but no palpable skin lesions, other signs or associated symptoms. (Figure 1) Otoscopy was normal with normal hearing thresholds on the right and mild conductive hearing loss on the left on pure tone audiometry. The rest of the physical examination and blood laboratory tests were unremarkable.

A temporal bone $\mathrm{CT}$ scan (to determine mass extent and the best site for repeat incisional biopsy) showed an intensely enhancing external ear mass extending to the outer cartilaginous portion of the auditory canal with multiple sub-centimeter enhancing nodules in the right parotid gland. (Figure 2) An excision biopsy between the junction of the mass and grossly normal-looking tissue of the right helix revealed Atypical Round Cell Tumor, subsequently diagnosed as T-cell Lymphoma after strongly staining with CD3 and Ki67 immunohistochemistry studies.

After unremarkable repeat chest $\mathrm{x}$-ray and abdominal $\mathrm{CT}$ findings, the patient underwent six cycles of chemotherapy using the CHOP (Doxorubicin, Vincristine, Cyclophosphamide, Prednisone) protocol for Peripheral T-cell lymphoma, not otherwise specified (PTCL-NOS), Stage II. Significant decrease in size of the auricular mass was noted from the second cycle until no mass was evident after completion of the regimen. No recurrence was noted during a 48-month followup period. (Figure 3 )
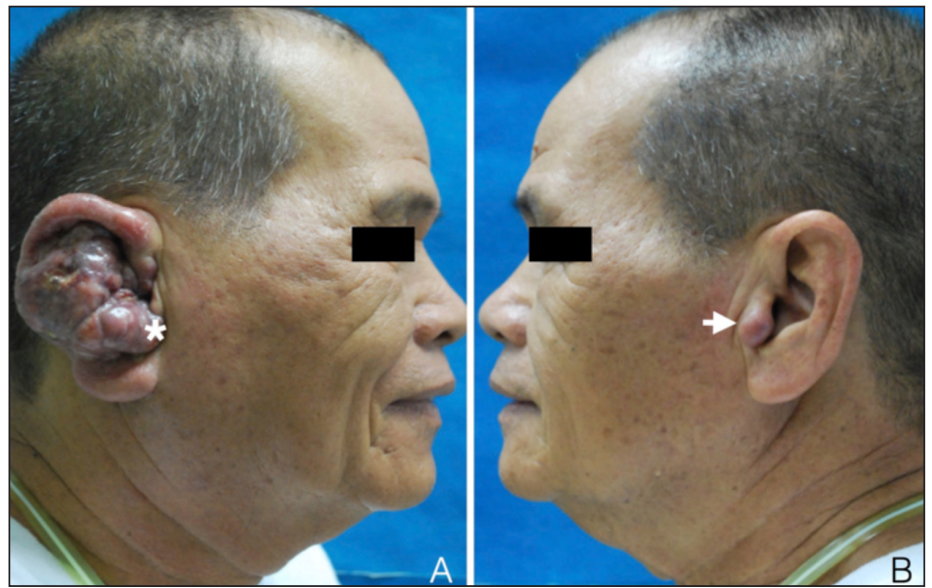

Figure 1. External ears on presentation A. Right nodular, non-tender auricular mass almost completely occupying the entire pinna and anterior cartilaginous portion of the external auditory canal (asterisk*); and B. Enlarged left tragus (arrow). 

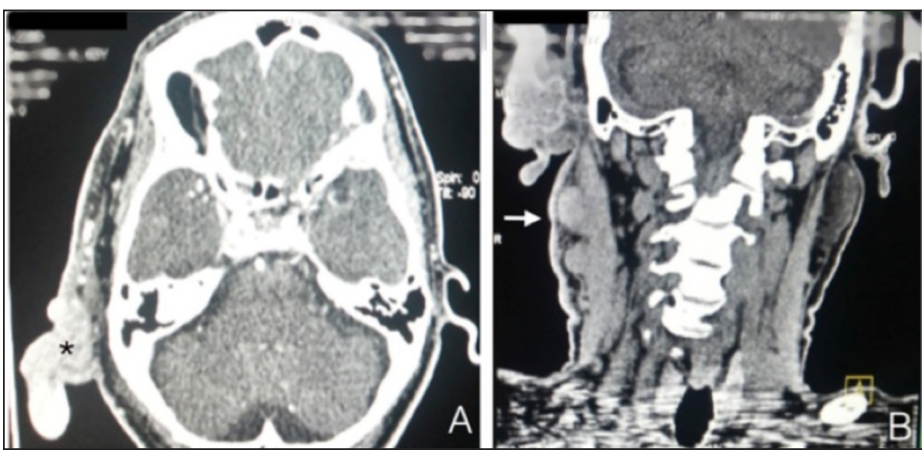

Figure 2. Temporal bone and neck contrast CT imaging A. Axial view showing intensely-enhancing right auricular mass with ill-defined borders extending to surrounding soft tissues (asterisk*); B. Coronal view showing enhancing right auricular mass extending to peri-parotid region with multiple enlarged right intra-parotid and cervical lymph nodes (Arrow).
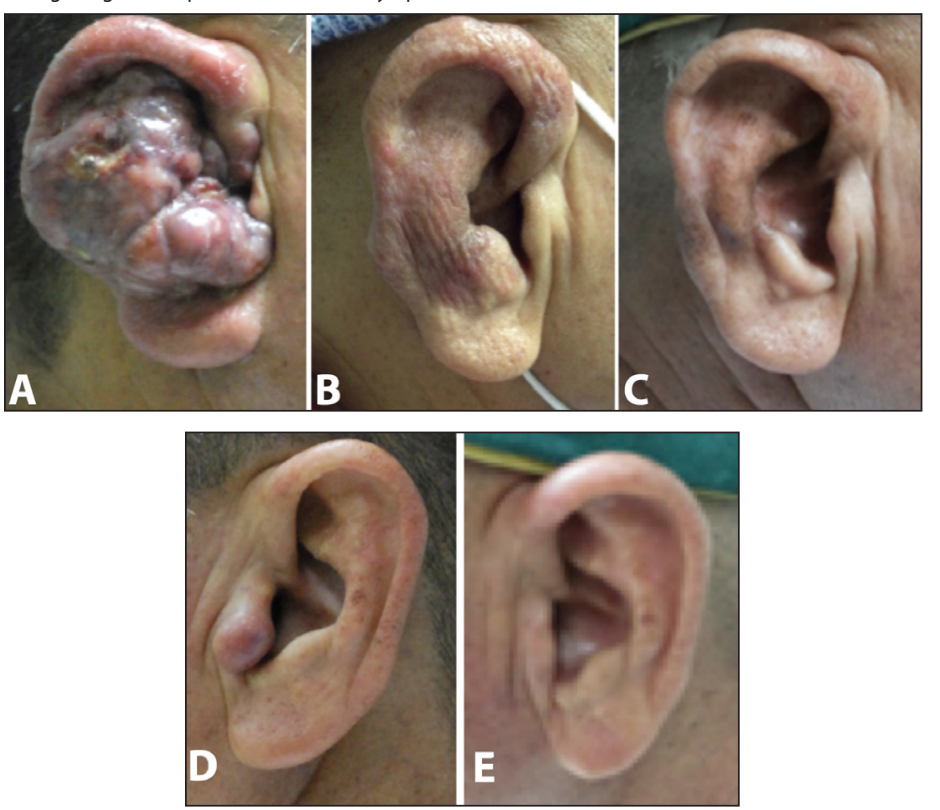

Figure 3. Response to CHOP treatment A. Right auricle before chemotherapy B. After 2nd cycle C. After 6th cycle D. Left ear before chemotherapy E. After 6th cycle of chemotherapy.

\section{DISCUSSION}

Because the external ear and auditory canal can be affected by various organisms especially in elderly, diabetic, and immunocompromised individuals, aggressive medical treatment is often warranted. A neoplastic etiology should be suspected when rapidly progressive ear deformities arise in cases of perichondritis and other non-infectious inflammatory conditions that are nonresponsive even to culture-guided therapy. In the absence of trauma, such other causes of ear deformity as sarcoidosis, perniosis, polychondritis and auricular pseudocyst must be carefully investigated by adequate biopsies and histopathologic studies to rule out malignant processes. ${ }^{1,2}$

Lymphomas represent approximately $2.5 \%$ of head and neck malignancies, and the majority present with cervical lymph node involvement. ${ }^{2}$ While $40 \%$ of head and neck lymphomas occur in such extranodal sites as the nasopharynx, lacrimal sac, temporal bone, or salivary glands, they rarely involve the auricle and external auditory canal. ${ }^{2}$ More common malignancies that may lead to auricular masses and deformities such as squamous cell carcinoma, adenocarcinoma, adenoid cystic carcinoma, mucoepidermoid carcinoma, basal cell carcinoma and rhabdomyosarcoma, must be ruled out., ${ }^{3,4}$ These conditions have distinct presentations and may arise from the external ear, middle ear or temporal bone before affecting the external auditory canal. ${ }^{5}$

The literature on extra-nodal lymphomas is scarce, with B- cell origin more commonly reported. On the other hand, peripheral T-cell lymphomas (PTCL) represent only 10-15\% of non-Hodgkin lymphoma, categorized as nodal, extra nodal or leukemic. ${ }^{6}$ A subtype of PTCL that do not correspond to any of the specifically defined T-cell entities in the World Health Organization (WHO) classification are defined as not otherwise specified PCTL (PTCL-NOS) ${ }^{7,8}$ However, based on a search of PubMed and HERDIN using the search terms "peripheral T-cell lymphoma," "auricle," and "external ear," to the best of our knowledge, PTCL-NOS has not been locally reported as primarily affecting the external ear. ${ }^{6,8}$

The clinical presentation of primary lymphomas of the EAC is nonspecific and they can be easily misdiagnosed and treated as infectious or inflammatory conditions of the external ear. As with other conditions, early and accurate diagnosis based on good clinical correlation with imaging studies must be achieved to allow early and specific treatment. For PTCL-NOS, the Ann Arbor staging system still applies although it was originally designed for Hodgkin lymphoma.' Surgical management has been reported for isolated auricular lymphomas but disseminated disease or involvement of complex structures such as the external ear warrant chemotherapy as the primary and definitive treatment. ${ }^{2}$ External beam radiation has likewise been reported as an option but may not be applicable in this particular case where structural preservation of the auricle is considered. ${ }^{2}$ To date, no therapeutic guidelines have been established due to the paucity of cases.

This case of PTCL-NOS of the auricle, just like other reported cases of lymphoma arising from the external auditory canal, appear to respond well with the standard CHOP regimen. The favorable resolution in our case suggests that surgical resection of the auricle should be reserved for non-response to standard treatment for lymphoma.

\section{REFERENCES}

1. Kindem S, Traves V, Requena C, Alcalá R, Llombart B, Serra-Guillén C, et al. Bilateral cauliflower ear as the presenting sign of B-cell chronic lymphocytic leukemia. J Cutan Pathol. 2014 Feb; 41(2): 73-77. DOI: 10.1111/cup.12290; PMID: 24460879

2. Bruschini L, De Vito A, Fortunato S, Pelosini M, Cervetti G, Petrini M, et al. A Case of Primary NonHodgkin's Lymphoma of the External Auditory Canal. Case Rep Otolaryngol. 2013; 2013: 138397. DOI:10.1155/2013/138397; PMID: 23984144; PMCID: PMC3747615

3. Merkus P, Copper MP, van Oers MH, Schouwenburg PF. Lymphoma in the ear. ORL J Otorhinolaryngol Relat Spec. 2000 Sep-Oct; 62(5): 274-77. DOI: 27759; PMID: 10965264.

4. González Delgado A, Argudo Marco F, Sánchez Martínez N, Sprekelsen Gassó C. T cell Non Hodgkin's lymphoma of the external auditory canal. Acta Otorrinolaringol Esp. 2008 Apr; 59(4):200-201. PMID: 18447981.

5. National Cancer Institute [Internet]. Cancer facts. Head and neck cancer: questions and answers [cited 2017 Feb 4]. Available from: http://www.cancer.gov/cancertopics/factsheet/Sites-Types/ head-and-neck/.

6. Shuto J, Ueyama T, Suzuki M, Mogi G. Primary lymphoma of bilateral external auditory canals. Am J Otolaryngol. 2002 Jan-Feb; 23(1):49-52. PMID: 11791249.

7. Lister TA, Crowther D, Sutcliffe SB, Glatstein E, Canellos GP, Young RC, et al. Report of a committee convened to discuss the evaluation and staging of patients with Hodgkin's disease: Cotswolds meeting. J Clin Oncol. 1989 Nov; 7(11): 1630-6. DOI: 10.1200/JCO.1989.7.11.1630; PMID: 2809679.

8. Peripheral T-Cell Lymphoma Facts [Internet]. White Plains, NY: Leukemia and lymphoma society. [Revised 2014 Jul; cited 2017 Feb 4]. Available from: http://www.cancer.gov/cancertopics/ factsheet/Sites-Types/head-and-neck/. 